\title{
Coherent bremsstrahlung in a bent crystal
}

\author{
M.V. Bondarenco* \\ Kharkov Institute of Physics and Technology, 1 Academic St., 61108 Kharkov, Ukraine.
}

(Dated: November 2, 2018)

\begin{abstract}
Coherent radiation spectrum from high energy $e^{ \pm}$in a bent crystal with arbitrary curvature distribution along the longitudinal coordinate is evaluated, based on the stationary phase approximation. For a uniformly bent crystal a closed-form expression for the spectrum is derived. The spectrum features include a dip at its beginning and the sharp end, which may split into two breaks depending on the particle incidence angle. Estimates of non-dipole radiation and multiple scattering effects are given. The value for the crystal bending angle at which the dipole coherent bremsstrahlung theory holds best appears to be $\sim 10^{-4} \mathrm{rad}$.
\end{abstract}

PACS numbers: 34.80.Pa, 29.27.-a, 41.60.-m, 78.70.-g

Keywords: bent crystal, coherent gamma-radiation, multiple scattering

\section{INTRODUCTION}

Gamma-radiation emitted by electrons and positrons at their non-channeled passage through planarly oriented bent crystals has been investigated in a few recent experiments [1, 2] searching for signatures of the charged particle volume reflection effect [3] in the radiation spectrum. However, the spectra observed were largely monotonous at typical photon frequencies $\omega$, and the difference between the measured spectra from positrons and electrons was basically of order of the experimental errors. At first sight, that may appear surprising, since the inherent dynamics and the final reflection angle of volume reflection itself are known to be sufficiently different in cases of positively and negatively charged particles.

In article [4] dedicated to computer simulation of the conditions of experiment [1] it was mentioned that the radiation spectrum must contain a component of so-called coherent bremsstrahlung in a bent crystal (CBBC) (see [5], appendix), arising at fast charged particle highly over-barrier motion, when perturbative treatment of particle interaction with the crystal is valid. Actually, radiation of that type may prove even dominant when the active crystallographic plane bending angle is many times larger than the critical value $\theta_{c}[20]$ - then the particle must spend most of its time traveling at angles to atomic planes much larger than critical, i. e., flying high above the potential barrier. Thereat, the frequency of the radiation emitted by the particle at a given instant is proportional to the local frequency of atomic plane crossing by the particle, as in ordinary coherent bremsstrahlung [6]. In course of the particle passage, the angle of atomic plane crossing varies, and the coherent radiation intensity accordingly sweeps over the spectrum. Close to the volume reflection point, of course, the particle motion will become non-perturbative, but the radiation from that region contributes relatively little to the spectrum as a whole, in contrast to the situation with the elastic scat-

*Electronic address: bon@kipt.kharkov.ua tering, where it is only the volume reflection point vicinity that contributes to the particle final deflection angle. The origin of the difference between the cases is obvious: the magnitude of the intra-crystalline transverse force is about the same all over the crystal, so all traversed crystal regions contribute commensurably to the total irradiation energy. As for the particle deflection angle, it is sensitive to the force sign, and thus receives little contribution from the regions where the force oscillates rapidly, as it does at large angles of atomic plane crossing.

In view of the described situation, prior to studies of radiation features stemming from non-perturbative segments of particle motion in bent crystals, it seems expedient to determine the shape of perturbative CBBC spectrum, which yields a wide continuous background and at the same time provides the conceptually simplest approximation. Unfortunately, so far it has not been evaluated in a form suitable for comparison with experiment. It is the purpose of this article to present a full, though basic calculation, and also to determine characteristic scales for the physics of the process. Last not least, we will assess robustness of the simplest CBBC theory against various deteriorating effects present in nature, such as multiple scattering and the dipole regime failure. It turns out that the range of the dipole CBBC theory is rather limited, although non-vanishing.

In view of the universality of the $\mathrm{CBBC}$ radiation in bent crystals of various shapes (existing examples include cylindrically bent crystals, sine-shaped bent crystals [7], other microfabricated configurations may appear in future), we extend our treatment to the case of arbitrary crystal bend profile. Our ability to cope with it grounds on the applicability of stationary phase approximation allowing one to treat the crystal curvature as locally constant. That variant of the stationary phase approximation is of different origin than the one arising in problems of synchrotron-like radiation, and does not contradict to the use of the dipole approximation in radiation.

The paper is organized as follows. In Sec. II we define the bent crystal planar continuous potential and the corresponding transverse force. In Sec. [II] we proceed to evaluating the particle deflection angle by such a force 


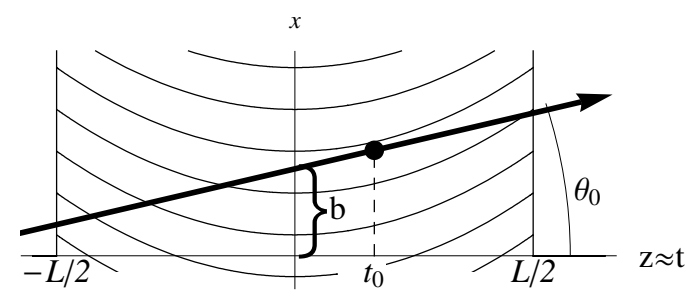

FIG. 1: Schematic of an ultra-high-energy particle passage through a thin bent crystal. The vicinity of point $t_{0}$ of the trajectory tangency to crystal bent planes gives the main contribution to the particle deflection angle.

to the leading order of high-energy perturbation theory of classical mechanics. In Sec. IV we evaluate the radiation spectrum in the dipole approximation, including the quantum effect of radiation recoil (allowing for photon energies to be of order of the initial electron's). In Sec.V the conditions of applicability of such an approximation are analyzed. Sec. VI provides a summary.

\section{PLANAR CONTINUOUS POTENTIAL IN A WEAKLY BENT CRYSTAL}

\section{A. Geometry definition}

At practice, for the coherent bremsstrahlung at overbarrier particle passage not to be spoiled by the particle multiple scattering on the target nuclei it is desirable to work with a crystal not thicker than a few millimeters (the same situation as for coherent bremsstrahlung in straight crystals). There are various techniques for manufacturing such crystals with bent atomic planes along the short direction, but basically they fall into two categories. First - one of the crystal transverse dimensions is made short, and even shorter than longitudinal (i. e., $\ll 1 \mathrm{~mm}$ ), which permits to bend the crystal along the longitudinal direction [8]. Second - both transverse dimensions are made sufficiently sizeable and the crystal so obtained is bent by some of the large dimensions, but securing that (short) crystallographic planes in the crystal, along which the beam is to be sent, acquire some bending, too. Specifically, the bending of the latter may be achieved through the action of anti-clastic forces, when the crystal is deformed simultaneously by both large transverse dimensions with different strength [9, 10] [21], or one might just arrange the active planes to be under some angle $\alpha$ to the large face, and then they must bend along with the large face, although $\sin \alpha$ times weaker. At the same time, for issues of particle passage through the crystal the deviations of the large crystal faces from planes may be neglected.

In any case, for what concerns description of the particle passage, the geometry implies particle incidence at some (small) angle $\theta_{0}$ to $z$-axis, chosen normal to the crystal large faces (let the latter be located at positions $z \approx-L / 2$ and $z \approx L / 2$ ), and the particle essentially interacts with the continuous potential of the planes depending only on single coordinate $x$ (see Fig. 1). The distance between the bent planes is practically unaffected by the crystal curvature, and the equation defining each plane takes the form

$$
x_{\mathrm{pl}}(z)=C_{\mathrm{pl}}+\xi(z),
$$

constants $C_{\mathrm{pl}}$ being equal-spaced with the inter-planar distance $d$.

Then, if the continuous inter-planar potential in the bent crystal was $V_{\text {straight }}(x)$ (a periodic function with period $d$ ), which corresponds to an acting force $F_{\text {straight }}(x)=-\frac{\partial V_{\text {straight }}}{\partial x}$, after bending of this crystal the force will modify to

$$
F(x, z)=F_{\text {straight }}(x-\xi(z))
$$

(still, it can be regarded as directed along $x$ ). For crystals of constant curvature [22],

$$
\xi(z) \approx \frac{z^{2}}{2 R}
$$

with $R=$ const being the atomic plane bending radius. In what follows, we will rely on the stationary phase approximation, in which the crystal curvature is treated locally, and is described by the local bending radius

$$
R(z)=\frac{1}{\left|\xi^{\prime \prime}(z)\right|},
$$

to emerge naturally in the following.

\section{B. Nearly parabolic continuous potential and the corresponding force}

The dynamics of a high-energy particle in a crystal may be described by ultra-relativistic classical mechanics [11]. As we have agreed, we will use perturbative description of particle interaction with the crystal; this is a rather common approach in the theory of coherent bremsstrahlung. Conditions thereof will be specified later (Sec. VV).

In the perturbative treatment of classical particle passage dynamics, as well as in quantum theory, it is advantageous to express the periodic continuous potential in a form of Fourier series. Such a representation is economic (provided only a few lowest harmonics dominate), and at the same time convenient when proceeding from description of a straight crystal to a bent one. For evaluation of the particle trajectory and the emitted radiation, of direct relevance is not the potential but the force acting on the particle. To define the force - firstly, in a straight crystal - it is convenient to choose the origin of $x$-axis in the middle of some inter-plane interval, with respect to which the potential is an even function of $x$, whereby the 
force has to be odd. Then, Fourier decomposition of the force involves sine functions only:

$$
F_{\text {straight }}(x)=\frac{2}{\pi} \sum_{n=1}^{\infty} F_{n}(-1)^{n} \sin \frac{2 \pi n x}{d}
$$

(the numerical factors have been introduced for further convenience).

In the simplest but important case of (110) planar orientation of a crystal with diamond-type lattice (e. g., silicon), the inter-planar continuous potential is approximable by a parabola, and the corresponding force - by a linear-sawtooth function, whose Fourier decomposition reads

$$
\begin{aligned}
F_{\text {cool }}^{(110)}(x) & =-\left.\frac{2 F_{1}}{d} x\right|_{|x|<d / 2}+\text { period. } \\
& =\frac{2 F_{1}}{\pi} \sum_{n=1}^{\infty} \frac{(-1)^{n}}{n} \sin \frac{2 \pi n x}{d} .
\end{aligned}
$$

According to (4a), $F_{1}$ has the meaning of the force extremal value achieved at $x \rightarrow-\frac{d}{2}+0$, the sign of $F_{1}$ being equal to that of the particle charge. On the contrary, in another important case of orientation (111), there are two different (but also nearly parabolic) wells within the period of the continuous potential [12], and the force Fourier decomposition turns somewhat more complicated:

$$
\begin{aligned}
& F_{\text {cool }}^{(111)}(x)=\frac{32}{\pi d} \sum_{n=1}^{\infty} \frac{(-1)^{n}}{n} \sin \frac{2 \pi n x}{d} \\
& \cdot\left\{\left(\frac{V_{L}}{3}+V_{S}\right) \cos \frac{\pi n}{4}-\frac{4}{\pi n}\left((-1)^{n} \frac{V_{L}}{9}+V_{S}\right) \sin \frac{\pi n}{4}\right\}
\end{aligned}
$$

( $V_{L}$ and $V_{S}$ have meaning of depths of the alternate unequal potential wells, while the well widths are exactly $d_{L}=\frac{3}{4} d$ and $\left.d_{S}=\frac{1}{4} d\right)$. Anyway, once one factors out here the value of the first Fourier coefficient,

$$
\left(\frac{V_{L}}{3}+V_{S}\right) \cos \frac{\pi}{4}+\frac{4}{\pi}\left(\frac{V_{L}}{9}-V_{S}\right) \sin \frac{\pi}{4}:=\frac{d}{16} F_{1},
$$

Eq. (5) will assume the form similar to (4b):

$$
F_{\text {cool }}^{(111)}(x)=\frac{2 F_{1}}{\pi} \sum_{n=1}^{\infty} \frac{(-1)^{n} c_{n}}{n} \sin \frac{2 \pi n x}{d} .
$$

Here $c_{n}$ is a sequence of coefficients of order unity, neither increasing nor decreasing as $n \rightarrow \infty$, and by definition $c_{1}=1$. 23]

To take into account thermal smearing of the potential, i. e., the force continuity at the locations of atomic planes, the simplest though heuristic trick is to increase the power of $n$ in the overall $\frac{1}{n}$ factor of the trigonometric series:

$$
\begin{gathered}
F_{\text {therm }}(x)=\frac{2 F_{1}}{\pi} \sum_{n=1}^{\infty} \frac{(-1)^{n} c_{n}}{n^{1+\epsilon}} \sin \frac{2 \pi n x}{d}, \\
\epsilon=\epsilon(T)>0 .
\end{gathered}
$$

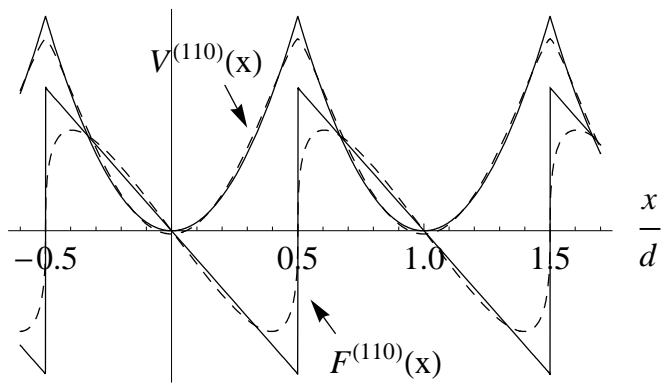

FIG. 2: Inter-planar continuous potential and the corresponding force (Eq. (8)) shapes (in arbitrary units) for positively charged particles in a silicon crystals with (110) orientation. Solid lines: $\epsilon=0$ (cooled crystal, Eq. (4a)); dashed lines: $\epsilon=0.4$ (room-temperature crystal, Eq. (8)). For negatively charged particles the signs of the functions reverse.

At that, the sequence $c_{n}$ (or its parameters $V_{L}, V_{S}$ ) may need to be corrected, but still the series is dominated by the first term, for which $c_{1} \approx 1$. Such a modification acts similarly to the conventional Debye-Waller exponential factor (which, in principle, is also heuristic, only its first order Maclaurin term being rigorously related to thermal averages). We refrain here from discussing the exact relation of $\epsilon$ with temperature $T$, only indicate that for the case of $\mathrm{Si}(110)$ at room temperature agreement with the potentials used in the literature is achieved at $\epsilon \approx 0.4$ (see Fig. 22), whereas for $\mathrm{Si}$ (111) it takes $\epsilon \sim 1$. To supply more motivation to our ansatz, note that in what follows the summation of series of the type (8) with constant $\epsilon$ and simple $c_{n}$ will yield Riemann $\zeta$-related functions. Such functions emerge as well for a zero-temperature potential $(\epsilon=0)$, only in the latter case the function arguments being integer or half-integer. Our approach corresponds to extension of those arguments to arbitrary fractional values, i. e. to an "analytic continuation", in order to model the effect of the temperature in a simplest way. None of the following numerical results (serving as estimates) depends crucially on this technique.

Practical bent crystals are usually manufactured from silicon. The relevant physical parameters for silicon are

$$
\begin{array}{lll}
d=1.9 \AA, & \left|F_{1}\right| \approx 6 \frac{\mathrm{GeV}}{\mathrm{cm}}, & (\mathrm{Si}(110)) \\
d=3.1 \AA, & \left|F_{1}\right| \approx 4 \frac{\mathrm{GeV}}{\mathrm{cm}} . & (\mathrm{Si}(111))
\end{array}
$$

Note that product $\left|F_{1}\right| d$ for those cases has practically identical values, which is important for the subsequent numerical estimates. But all our figures will be drawn only for a simpler case (110).

\section{INFINITESIMAL DEFLECTION ANGLE}

In this section we shall analyze the elastic scattering of ultra-high energy particles by the continuous force defined in the previous section. Choose the time reference 
point at the moment of the particle passage through the middle of the crystal $(z=0)$, so that we may equate $t \simeq z$ (we will use units $c=\hbar=1$ ). Since the beam width in practice is always greater than the inter-planar distance, there is essentially a uniform distribution of particles in transverse impact parameters. Defining impact parameter $b$ of an individual particle as the trajectory initial asymptote intercept on $x$-axis, i. e. at $z=0$ (see Fig. 1), the force acting on the particle in a bent crystal can be written as

$$
\begin{aligned}
F(t)= & \Theta\left(\frac{L}{2}-|t|\right) \frac{2 F_{1}}{\pi} \\
& \cdot \sum_{n=1}^{\infty} \frac{(-1)^{n}}{n^{1+\epsilon}} \sin \left(2 \pi n \frac{b+\theta_{0} t-\xi(t)}{d}\right),
\end{aligned}
$$

with $\Theta(v)$ - the Heavyside unit step function (zero for negative arguments and unity for positive ones).

First of all, let us evaluate the particle deflection angle. Asymptotically, to leading order in the potential to energy ratio $V / E$, the deflection angle is proportional to the integral of force (10) along the particle unperturbed straight path [24]:

$$
\begin{aligned}
\theta_{\text {Born }}\left(\theta_{0}, b\right)= & \frac{1}{E} \int_{-L / 2}^{L / 2} F(t) d t \\
= & \frac{2}{\pi R_{c}} \sum_{n=1}^{\infty} \frac{(-1)^{n} c_{n}}{n^{1+\epsilon}} \\
& \cdot \int_{-L / 2}^{L / 2} \sin \left(2 \pi n \frac{b+\theta_{0} t-\xi(t)}{d}\right) d t
\end{aligned}
$$

where

$$
R_{c}=E / F_{1}
$$

is the Tsyganov critical radius [13] (the above definition of $R_{c}$ yet allows it, along with $F_{1}$, to have different sign depending on the particle charge sign). If the crystal bending is macroscopic, in the sense that displacement $\xi$ of the planes is (generally) $\gg d$, the integrand is rapidly oscillatory. For evaluation of such an integral, one may employ the stationary phase approximation [14]. This requires, in the first place, finding stationary phase points $t_{s}$ at which

$$
t_{s}\left(\theta_{0}\right): \quad \theta_{0}-\left.\frac{d \xi}{d t}\right|_{t=t_{s}}=0
$$

i. e., the points of tangency of a ray with slope $\theta_{0}$ to the family of bent crystalline planes. For a convex function $\xi$ such a point is unique - and for simplicity we will assume this to be the case, dubbing it $t_{0}$ (see Fig. 11). Then, expanding function $\xi(t)$ in Taylor series about $t_{0}$ up to

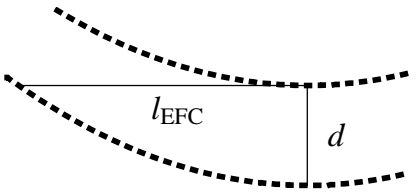

FIG. 3: Geometric interpretation of the coherence length of a fast particle traversing a bent crystal: $l_{\mathrm{EFC}}$ is a half-chord within a curved crystalline plane (radius $R$ ) tangential to the next curved plane at distance $d$ from the initial one.

quadratic terms, one brings (11) to the form

$$
\begin{array}{r}
\theta_{\text {Born }}\left(\theta_{0}, b\right) \approx \frac{2}{\pi R_{c}} \sum_{n=1}^{\infty} \frac{(-1)^{n} c_{n}}{n^{1+\epsilon}} \\
\cdot \Im \mathfrak{m} \int_{-L / 2}^{L / 2} d t \exp \left\{i \frac { 2 \pi n } { d } \left(b+\theta_{0} t_{0}-\xi\left(t_{0}\right)\right.\right. \\
\left.\left.-\frac{1}{2} \xi^{\prime \prime}\left(t_{0}\right)\left(t-t_{0}\right)^{2}\right)\right\} .
\end{array}
$$

Now, if point $t_{0}$ belongs to the interval $-\frac{L}{2}<t_{0}<\frac{L}{2}$, the integral converges in a small vicinity of this point of the width

$$
\left|t-t_{0}\right| \sim l_{\mathrm{EFC}}\left(t_{0}\right)=\sqrt{2 R\left(t_{0}\left(\theta_{0}\right)\right) d},
$$

where

$$
R(t)=\frac{1}{\left|\xi^{\prime \prime}(t)\right|}
$$

(for a geometric construction see Fig. 3). Physically, this is the length on which the deflecting external field acts periodically, and will be referred to as the external field coherence (EFC) length. Then, the integration limits in (14) may as well be extended to infinity, and the integral evaluates by a standard formula

$$
\int_{-\infty}^{\infty} e^{i A t^{2}} d t=e^{i \frac{\pi}{4} \operatorname{sgn} A} \sqrt{\frac{\pi}{|A|}}, \quad(\Im \mathfrak{m} A=0)
$$

with $\operatorname{sgn}$ function defined as $\operatorname{sgn} A=-1(+1)$ if $A<$ $0(A>0)$. If, on the contrary, $t_{0}$ falls beyond the integration interval, the integrand is everywhere rapidly oscillatory, and the result is small (yet there are contributions from the end-points, inferior to those from stationary phase points, but they will be neglected throughout for simplicity). With this accuracy,

$$
\begin{array}{r}
\theta_{\text {Born }}\left(\theta_{0}, b\right) \approx \Theta\left(\frac{L}{2}-\left|t_{0}\left(\theta_{0}\right)\right|\right) \frac{\sqrt{R\left(t_{0}\left(\theta_{0}\right)\right) d}}{R_{c}} \\
\cdot \frac{2}{\pi} \sum_{n=1}^{\infty} \frac{(-1)^{n} c_{n}}{n^{3 / 2+\epsilon}} \sin \left(-\frac{\pi}{4} \operatorname{sgn} \xi^{\prime \prime}\left(t_{0}\left(\theta_{0}\right)\right)\right. \\
\left.+2 \pi n\left[\frac{b+\theta_{0} t_{0}\left(\theta_{0}\right)-\xi\left(t_{0}\left(\theta_{0}\right)\right)}{d}\right]\right) .
\end{array}
$$


Hence, the magnitude of deflection angles is determined by the crystalline plane curvature radius in the point of the trajectory tangency to the bent planes.

As for functions $t_{0}\left(\theta_{0}\right), \xi\left(t_{0}\left(\theta_{0}\right)\right)$ appearing in (17), they may be obtained explicitly only if $\xi(t)$ is a sufficiently simple analytic function. For instance, in case of a crystal of constant curvature (3) they express through the only available parameter - the plane bending radius:

$$
\begin{gathered}
t_{0}=R \theta_{0}, \quad \xi\left(t_{0}\right)=\frac{R}{2} \theta_{0}^{2}, \quad \theta_{0} t_{0}-\xi\left(t_{0}\right)=\frac{R}{2} \theta_{0}^{2} . \\
(R=\text { const })
\end{gathered}
$$

So, at practice reversible analytic parameterizations of the bending profile $\xi(t)$ are favored.

For the case of orientation (110), when $c_{n} \equiv 1$, the sum in the right-hand side of (17) can be expressed in terms of Hurwitz (generalized Riemann) $\zeta$-functions:

$$
\begin{aligned}
\frac{2}{\pi} \sum_{n=1}^{\infty} \frac{(-1)^{n}}{n^{3 / 2+\epsilon}} \sin \left\{-\frac{\pi}{4} \operatorname{sgn} \xi^{\prime \prime}\left(t_{0}\right)+2 \pi n \beta\right\} \\
=\operatorname{sgn} \xi^{\prime \prime}\left(t_{0}\right) \frac{2(2 \pi)^{\frac{1}{2}+\epsilon}}{\Gamma\left(\frac{3}{2}+\epsilon\right)} \\
\cdot\left(\cos \frac{\pi \epsilon}{2} \zeta\left(-\frac{1}{2}-\epsilon,\left\{\frac{1}{2}+\beta \operatorname{sgn} \xi^{\prime \prime}\left(t_{0}\right)\right\}_{\mathrm{f}}\right)\right. \\
\left.-\sin \frac{\pi \epsilon}{2} \zeta\left(-\frac{1}{2}-\epsilon,\left\{\frac{1}{2}-\beta \operatorname{sgn} \xi^{\prime \prime}\left(t_{0}\right)\right\}_{\mathrm{f}}\right)\right),
\end{aligned}
$$

where

$$
\beta\left(\frac{b}{d}, \theta_{0}\right)=\frac{b+\theta_{0} t_{0}\left(\theta_{0}\right)-\xi\left(t_{0}\left(\theta_{0}\right)\right)}{d}
$$

characterizes the impact parameter of an oblique trajectory relative to the bent planes in point $z=t_{0}$, and $\zeta(\alpha, v)$ is the Hurwitz zeta-function [15], while braces $\{\ldots\}_{\mathrm{f}}$ in its second argument indicate the entry fractional part (ranging from 0 to 1 ). For orientation (111) the result can be expressed through Hurwitz zeta-functions in a similar manner, but it is somewhat more bulky and shall not be quoted herein.

Function (18) (shown in Fig. (4) is not particularly sensitive to the value of $\epsilon$, except around the fracture points. The latter ones are located at

$$
\beta= \pm \frac{1}{2}, \pm \frac{3}{2}, \ldots
$$

In those points, function (18) (and therewith (17)) is extremal and achieves the value

$$
\begin{aligned}
\max _{b} \frac{\theta_{\mathrm{Born}}\left(\theta_{0}, b\right)}{\operatorname{sgn} \xi^{\prime \prime}}=- & \Theta\left(\frac{L}{2}-\left|t_{0}\left(\theta_{0}\right)\right|\right) \\
& \zeta\left(\frac{3}{2}+\epsilon\right) \frac{\sqrt{2 R\left(t_{0}\left(\theta_{0}\right)\right) d}}{\pi R_{c}},
\end{aligned}
$$

where $\zeta(\alpha)=\sum_{n=1}^{\infty} n^{-\alpha}$ is the ordinary Riemann zetafunction.

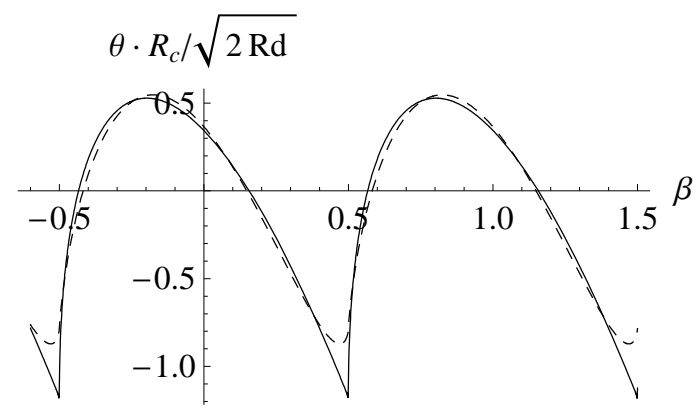

FIG. 4: Deflection angle of a positively charged particle in a bent $\mathrm{Si}(110)$ crystal, in units of $\frac{\sqrt{2 R d}}{R_{c}}$, vs. the impact parameter variable $\beta=\frac{b}{d}+$ const (see Eq. (19)). Solid line: $\epsilon=0$ (cooled crystal), dashed line: $\epsilon=0.4$ (room-temperature crystal).

The noticeable asymmetry of function(s) in Fig. 4 can be traced to the phase shift $\frac{\pi}{4}$, arising within the stationary phase approximation. The average value of the deflection angle over the impact parameters is strictly zero, as an average of a sum of sine functions over their full period. The physical reason behind that is the uniform distribution of an unperturbed particle flow over the crystal, entailing equal influence of positive and negative forces on the entire beam. So, in the adopted first order of perturbation theory there is no signature of volume reflection of the beam. Non-zero, however, is the angular spread acquired by the beam, whose measure is the deflection angle mean square:

$$
\begin{aligned}
\left\langle\theta_{\text {Born }}^{2}\right\rangle & =\frac{1}{d} \int_{0}^{d} d b \theta^{2}\left(\theta_{0}, b\right) \\
& \approx \frac{2 R\left(t_{0}\left(\theta_{0}\right)\right) d}{\pi^{2} R_{c}^{2}} \sum_{n=1}^{\infty} \frac{c_{n}^{2}}{n^{3+2 \epsilon}} .
\end{aligned}
$$

The latter quantity will also play part in the treatment of radiation.

\section{DIPOLE COHERENT BREMSSTRAHLUNG}

Having established in the previous section the description of the particle passage through the crystal, we are in a position to calculate the accompanying radiation. As is typical for bremsstrahlung from relativistic particles, the radiation is concentrated within a cone of angles $\sim \gamma^{-1}$ about the forward direction, and those small angles may be treated inclusively. Let $\omega$ stand for the photon frequency (energy). Within the dipole approximation, the spectrum of radiation integrated over emission angles, and averaged over the impact parameters $b$ of particles 
in the beam, expresses through the acting force as [6, 17]

$$
\begin{array}{r}
\frac{d E_{\mathrm{CBBC}}}{d \omega}=\frac{e^{2} E^{\prime} \omega}{2 \pi E^{3}} \int_{q_{\min }}^{\infty} \frac{d q}{q^{2}}\left(1+\frac{\omega^{2}}{2 E E^{\prime}}-\frac{2 q_{\min }}{q}+\frac{2 q_{\min }^{2}}{q^{2}}\right) \\
\cdot \frac{1}{d} \int_{0}^{d} d b\left|F\left(q, \theta_{0}, b\right)\right|^{2},
\end{array}
$$

where

$$
E^{\prime}=E-\omega, \quad q_{\min }=q_{\min }(\omega)=\frac{\omega m^{2}}{2 E E^{\prime}},
$$

(allowing for $\omega \sim E$, to account for quantum radiation recoil effects), and

$$
F\left(q, \theta_{0}, b\right)=\int_{-\infty}^{\infty} d t e^{i q t} F\left(t, \theta_{0}, b\right) .
$$

Thus, $q$ is a frequency of the force acting on the particle, and (24) is the Fourier transformation of the intra-crystal force.

With $F(t)$ given by Eq. (10), its Fourier transform is conveniently evaluated by decomposing the sine function into a pair of exponentials:

$$
\begin{array}{r}
F\left(q, \theta_{0}, b\right)=\frac{2 F_{1}}{\pi} \sum_{n=1}^{\infty} \frac{(-1)^{n} c_{n}}{n^{1+2 \epsilon}} \\
\cdot \int_{-L / 2}^{L / 2} d t e^{i q t} \sin \left(2 \pi n \frac{b+\theta_{0} t-\xi(t)}{d}\right) \\
=\frac{F_{1}}{\pi i} \sum_{n=1}^{\infty} \frac{(-1)^{n}}{n^{1+\epsilon}} \int_{-L / 2}^{L / 2} d t\left(e^{i\left(2 \pi n \frac{b+\theta_{0} t-\xi(t)}{d}+q t\right)}\right. \\
\left.-e^{-i\left(2 \pi n \frac{b+\theta_{0} t-\xi(t)}{d}-q t\right)}\right) .
\end{array}
$$

When averaging the square of (25) over the impact parameters, we employ the identity

$$
\frac{1}{d} \int_{0}^{d} d b e^{2 \pi i n \frac{b}{d}} e^{-2 \pi i m \frac{b}{d}}=\delta_{n m}
$$

by virtue of which

$$
\begin{aligned}
& \frac{1}{d} \int_{0}^{d} d b\left|F\left(q, \theta_{0}, b\right)\right|^{2} \\
&=\frac{F_{1}^{2}}{\pi^{2}} \sum_{n=1}^{\infty} \frac{c_{n}^{2}}{n^{2+2 \epsilon}}\left(\left|\int_{-L / 2}^{L / 2} d t e^{i\left(2 \pi n \frac{\theta_{0} t-\xi(t)}{d}+q t\right)}\right|^{2}\right. \\
&\left.+\left|\int_{-L / 2}^{L / 2} d t e^{i\left(2 \pi n \frac{\theta_{0} t-\xi(t)}{d}-q t\right)}\right|^{2}\right)
\end{aligned}
$$

(note the absence of interference between the exponents after the averaging).

Evaluation of each of the two oscillatory integrals in (27) is carried out by the stationary phase approximation, as in the previous section. The external field coherence length is the same as (15), only the location of

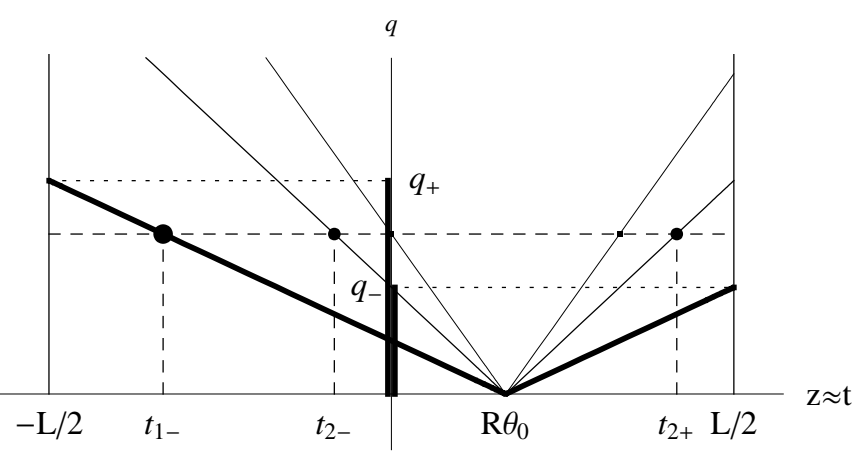

FIG. 5: Local frequencies of plane crossing (thick oblique lines), and the frequencies of higher harmonics (thinner oblique lines), as functions of the particle longitudinal coordinate (time). Drawn for a specific case of crystal with constant curvature, when $q(t)$ dependencies are linear. Vice versa, the construction may be used for determination of stationary phase points for a given frequency (dashed lines). Thick intercepts on the $q$-axis schematically show a double-step distribution of the force frequencies described by function $|F(q)|^{2}$ (see also Fig. 9r).

the stationary-phase point $t_{n \pm}$, about which function $\xi(t)$ has to be Taylor-expanded, now depends on $\theta_{0}$ and $q / n$. The equations for stationary phase points read

$$
t_{n \pm}\left(q, \theta_{0}\right): \quad \xi^{\prime}\left(t_{n \pm}\right)-\theta_{0}= \pm \frac{q d}{2 \pi n} .
$$

(In accord with (27), radiation from different stationary phase points does not interfere). Physically, $\xi^{\prime}-\theta_{0}$ represents the angle between the beam and the crystalline planes in the stationary phase point. Thereby, Eq. (28) may be viewed as a local coherent bremsstrahlung condition, in which the local frequency of the driving external force is proportional to the local frequency of crystalline plane crossing by the particle, which in turn is proportional to the local angle of the trajectory inclination to the planes (cf. [6]). Ultimately, the approximate $t$-integration gives

$$
\begin{aligned}
& \left|\int_{-L / 2}^{L / 2} d t e^{i\left(2 \pi n \frac{\theta_{0} t-\xi(t)}{d} \pm q t\right)}\right|^{2} \\
& \quad \approx \Theta\left(\frac{L}{2}-\left|t_{n \pm}\left(q, \theta_{0}\right)\right|\right) \frac{R\left(t_{n \pm}\left(q, \theta_{0}\right)\right) d}{n} .
\end{aligned}
$$

Substitution of (27, 29) to (22) leads to the result for the radiation spectrum of coherent bremsstrahlung in a 
bent crystal:

$$
\begin{aligned}
& \frac{d E_{\mathrm{CBBC}}}{d \omega} \approx \frac{e^{2} F_{1}^{2} d}{2 \pi^{3}} \frac{E^{\prime} \omega}{E^{3}} \\
& \cdot \sum_{n=1}^{\infty} \frac{c_{n}^{2}}{n^{3+2 \epsilon}} \int_{q_{\min }}^{\infty} \frac{d q}{q^{2}}\left(1+\frac{\omega^{2}}{2 E E^{\prime}}-\frac{2 q_{\min }}{q}+\frac{2 q_{\min }^{2}}{q^{2}}\right) \\
& \cdot\left\{\Theta\left(\frac{L}{2}-\left|t_{n+}\left(q, \theta_{0}\right)\right|\right) R\left(t_{n+}\left(q, \theta_{0}\right)\right)\right. \\
&\left.+\Theta\left(\frac{L}{2}-\left|t_{n-}\left(q, \theta_{0}\right)\right|\right) R\left(t_{n-}\left(q, \theta_{0}\right)\right)\right\} .
\end{aligned}
$$

By virtue of the power factor $\frac{1}{n^{3+2 \epsilon}}$, this sum is strongly dominated by the term $n=1$, so the coherent bremsstrahlung spectrum shapes for crystal orientations (110) and (111) appear to be only marginally different, and the temperature effect on the coherent radiation is also small.

In what follows we will concentrate on application of formula (30) to crystals of constant curvature. In that case the solution to Eq. (28) is $t_{n \pm}=\left(\theta_{0} \pm \frac{q d}{2 \pi n}\right) R$ (see Fig. (5), and in (30) one may draw constant $R$ out of the integral, which allows one to accomplish the integration in terms of elementary functions. Introducing parameters

$$
q_{ \pm}=\frac{2 \pi}{d}\left(\frac{L}{2 R} \pm\left|\theta_{0}\right|\right)
$$

(signifying the frequencies of active crystalline plane crossing at the entrance and at the exit from the crystal) and a function

$$
\begin{aligned}
D\left(v, \frac{\omega}{E}\right)= & \int_{v}^{1} d y\left(1+\frac{\omega^{2}}{2 E E^{\prime}}-2 y+2 y^{2}\right) \\
= & (1-v)\left(\frac{2-v+2 v^{2}}{3}+\frac{\omega^{2}}{2 E E^{\prime}}\right) \\
\equiv & \frac{1}{3}+\frac{1}{2}\left(\frac{1}{2}-v\right)+\frac{2}{3}\left(\frac{1}{2}-v\right)^{3} \\
& +(1-v) \frac{\omega^{2}}{2 E E^{\prime}}
\end{aligned}
$$

$$
(0 \leq v \leq 1)
$$

the expression for the radiation spectrum assumes the form

$$
\begin{aligned}
& \quad \frac{d E_{\mathrm{CBBC}}}{d \omega}=\frac{e^{2} F_{1}^{2} R d}{\pi^{3} m^{2}} \frac{E^{\prime 2}}{E^{2}} \\
& \cdot \sum_{n=1}^{\infty} \frac{c_{n}^{2}}{n^{3+2 \epsilon}}\left\{\Theta\left(n q_{-}-q_{\text {min }}\right) D\left(\frac{q_{\text {min }}}{n q_{-}}, \frac{\omega}{E}\right)\right. \\
& +\Theta\left(n q_{-}+q_{\text {min }}\right) \Theta\left(n q_{+}-q_{\text {min }}\right) D\left(\frac{q_{\text {min }}}{n q_{+}}, \frac{\omega}{E}\right) \\
& \left.+\Theta\left(-n q_{-}-q_{\text {min }}\right)\left[D\left(\frac{q_{\text {min }}}{n q_{+}}, \frac{\omega}{E}\right)-D\left(\frac{q_{\text {min }}}{n\left|q_{-}\right|}, \frac{\omega}{E}\right)\right]\right\} .
\end{aligned}
$$

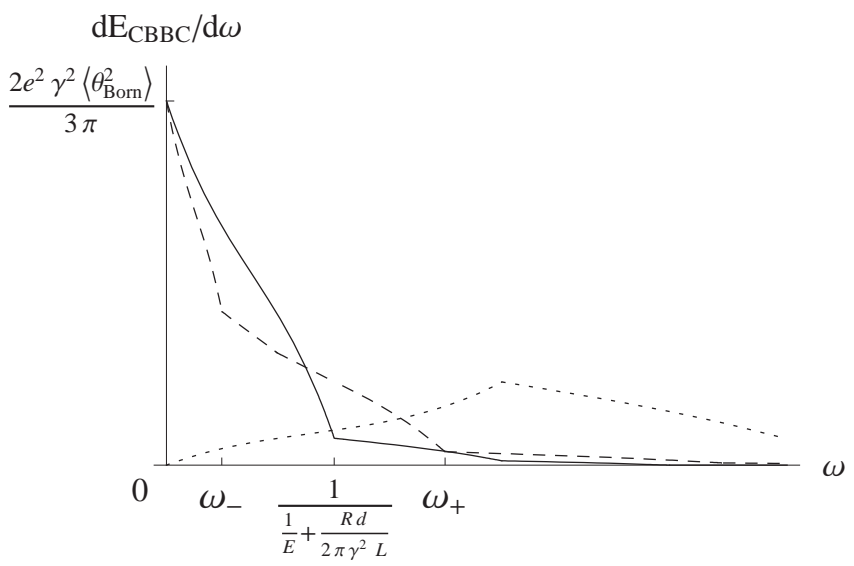

FIG. 6: Coherent radiation spectra (neglecting multiple scattering and incoherent bremstrahlung), for a fixed value of $\frac{2 \pi L \gamma^{2}}{R d} \ll E$ and several values of $\left|\theta_{0}\right|$. Solid line: $\theta_{0}=0$ ( $\omega_{-}$and $\omega_{+}$coincide); dashed: $\left|\theta_{0}\right|=\frac{L}{3 R}$; dotted: $\left|\theta_{0}\right|=\frac{L}{R}$. The impact of temperature effects on radiation is negligible; the type of crystal orientation ((110) or (111)) mainly affects the frequency and intensity scales.

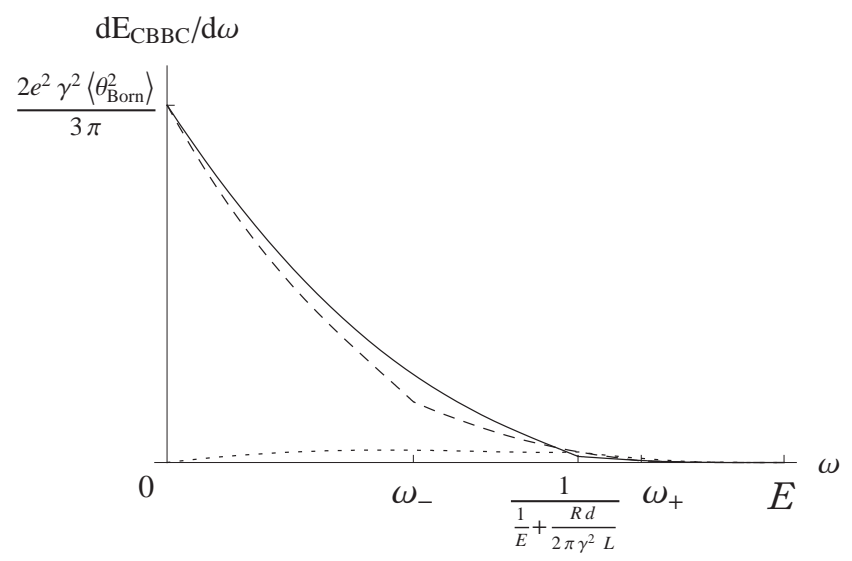

FIG. 7: Same as Fig. 6, for $\frac{2 \pi L \gamma^{2}}{R d}=2 E$. At this electron energy, the radiation spectrum shape is already strongly influenced by the quadratic factor $E^{\prime 2} / E^{2}$ in Eq. (33), obscuring the spectrum features.

The behavior of the spectrum for different incidence angles is illustrated in Figs. 6, 7 Despite being composed of discontinuous $\Theta$-functions, in total (33) is everywhere continuous, as is conditioned by its initial integral representation (30). Still, there are discontinuities in the derivative of (33) manifesting themselves as sharp curve breaks ("ankle"-type). Beyond the first two, major breaks [25] (corresponding to $n=1$ ) the spectrum effectively ends, and only contributions from higher harmonics remain. Those main "ankles" are located at photon energies 26

$$
\omega_{ \pm}=\frac{1}{\frac{1}{E}+\frac{1}{2 \gamma^{2} \mid q_{ \pm}}}
$$


Note that when $\left|\theta_{0}\right|$ is only slightly below $\frac{L}{2 R}$, then $\omega_{-} \ll \omega_{+}$, and at $\omega \leq \omega_{-}$the spectrum develops a sharp spike, although superimposed on the background of equal height. That condition corresponds to a trajectory nearly tangential to the crystalline planes at the entrance or exit from the crystal, and although this feature may be of experimental utility, one should beware that our present stationary phase approximation neglecting end-point effects, as well as the dipole approximation itself, are in substantial error there.

There are other important features of the CBBC spectrum concerning dependencies on the geometric parameters $R, L$ and $\theta_{0}$ :

(i) It is natural that the differential cross-section of coherent radiation is proportional to the square of the field strength and to the square of the coherence length (15). We accentuate that the coherence length in our problem is of external origin and independent of $\omega$ - that is a length on which the particle-crystal interaction may be regarded as periodic. In contrast, the photon formation length [27] $l_{\text {form }}=q_{\min }^{-1}(\omega) \gtrsim q_{ \pm}^{-1}$ depends on $\omega$. It sets the scale of resulting photon energies, correlating with spacings $q_{ \pm}^{-1}$ between the neighboring crystalline planes measured along the particle trajectory at the entrance to and at the exit from the crystal.

(ii) One can check that as $\omega \rightarrow 0$ the limit of (30) is

$$
\frac{d E_{\mathrm{CBBC}}}{d \omega} \underset{\omega \rightarrow 0}{\rightarrow} \frac{2 e^{2}}{3 \pi} \gamma^{2}\left\langle\theta_{\text {Born }}^{2}\right\rangle
$$

with $\left\langle\theta_{\text {Born }}^{2}\right\rangle$ given by Eq. (21). Apparently, this value does not depend on the crystal thickness $L$.

(iii) The total radiation energy emitted per one electron

$$
E_{\mathrm{CBBC}}=\int_{0}^{E} d \omega \frac{d E_{\mathrm{CBBC}}}{d \omega}
$$

expresses rather simply and quite differently in two limiting cases: when the photon recoil effects are negligible, and when they are crucial. If the "moderately high energy" condition $2 \gamma^{2} q_{+} \ll E$ is met, then $q_{\min } \approx \frac{\omega}{2 \gamma^{2}}$ and the second argument of all the $D$-functions in (33) may be put to zero. For this case, one finds

$$
\begin{gathered}
E_{\mathrm{CBBC}} \simeq L \frac{8 e^{2}}{3 \pi^{2}} \gamma^{2} \frac{F_{1}^{2}}{m^{2}} \sum_{n=1}^{\infty} \frac{c_{n}^{2}}{n^{2+2 \epsilon}} . \\
\left(\gamma \ll \frac{m d}{2 \pi} \frac{R}{L} \sim 10^{2} \frac{R}{L}\right)
\end{gathered}
$$

In contrast to the differential intensity, the total emitted energy here is proportional not to the square of the coherence length but to the crystal thickness. Remarkably, it does not depend on $R$, nor $\theta_{0}$, and just equals to the total energy of coherent bremsstrahlung radiation in a straight crystal of thickness $L$.

If the opposite condition $2 \gamma^{2} q_{+} \gg E$ is realized, then the first argument of all the $D$-functions in (33) may be put to zero, giving

$$
\begin{gathered}
\left.E_{\mathrm{CBBC}} \simeq \frac{d E_{\mathrm{CBBC}}}{d \omega}\right|_{\omega \rightarrow 0} \int_{0}^{E} d \omega \frac{E^{\prime 2}}{E^{2}}\left(1+\frac{3 \omega^{2}}{4 E E^{\prime}}\right) \\
=E \frac{19 e^{2}}{36 \pi^{3}} \frac{F_{1}^{2} R d}{m^{2}} \sum_{n=1}^{\infty} \frac{c_{n}^{2}}{n^{3+2 \epsilon}} \Theta\left(\frac{L}{2 R}-\left|\theta_{0}\right|\right) . \\
\left(\gamma \gg \frac{m d}{2 \pi} \frac{R}{L} \sim 10^{2} \frac{R}{L}\right)
\end{gathered}
$$

So, in this limit even the total radiation energy does not depend on the crystal thickness.

(iv) $\mathrm{At}$

$$
\left|\theta_{0}\right| \gg \frac{L}{2 R}
$$

(a large incidence angle or the straight crystal limit), Eqs. (31] 34) yield $q_{-} \simeq-q_{+}<0, \omega_{-} \simeq$ $\omega_{+}$. Then in (33) the term with $\Theta\left(n q_{-}-q_{\min }\right)$ vanishes. The next term containing $\Theta\left(n q_{-}+\right.$ $\left.q_{\text {min }}\right) \Theta\left(n q_{+}-q_{\text {min }}\right)$ is non-zero only in relatively small intervals $n\left|q_{-}\right| \leq q_{\min }<n q_{+}$, yet the corresponding $D$-function has its first argument close to unity and thereby is small (cf. Eq. (32a)):

$$
D\left(\frac{q_{\min }}{n q_{+}}, \frac{\omega}{E}\right) \simeq\left(1-\frac{q_{\min }}{n q_{+}}\right)\left(1+\frac{\omega^{2}}{2 E E^{\prime}}\right) \ll 1 .
$$

Lastly, the term containing $\Theta\left(-n q_{-}-q_{\min }\right)$ contributes on the entire interval $q_{\min } \leq n\left|q_{-}\right|$, i. e., basically at $\omega \leq \omega_{-} \simeq \frac{1}{\frac{1}{E}+\frac{d}{4 \pi \gamma^{2}\left|\theta_{0}\right|}}$, but there is a valuable cancelation between the corresponding $D$ functions:

$$
\begin{aligned}
& D\left(\frac{q_{\min }}{n q_{+}}, \frac{\omega}{E}\right)-D\left(\frac{q_{\min }}{n\left|q_{-}\right|}, \frac{\omega}{E}\right) \\
& \left.\simeq \frac{\partial}{\partial v} D\left(v, \frac{\omega}{E}\right)\right|_{v=\frac{q_{\min }}{n \mid q_{-}}} \frac{q_{\min }}{n}\left(\frac{1}{q_{+}}-\frac{1}{\left|q_{-}\right|}\right) \\
& \cong\left(1-2 \frac{q_{\min }}{n\left|q_{-}\right|}+2 \frac{q_{\min }^{2}}{n^{2}\left|q_{-}\right|^{2}}+\frac{\omega^{2}}{2 E E^{\prime}}\right) \frac{q_{\min }}{n q_{-}^{2}} \frac{2 \pi L}{R d}, \\
& \left|q_{-}\right| \simeq \frac{2 \pi\left|\theta_{0}\right|}{d} .
\end{aligned}
$$

Therewith, the radiation spectrum reduces to

$$
\begin{aligned}
& \frac{d E}{d \omega} \simeq L \frac{e^{2} F_{1}^{2} d^{2}}{2 \pi^{4} m^{2} \theta_{0}^{2}} \frac{E^{\prime 2}}{E^{2}} q_{\min } \\
& \cdot \sum_{n=1}^{\infty} \Theta\left(n-\frac{q_{\min }}{\left|q_{-}\right|}\right) \frac{c_{n}^{2}}{n^{4+2 \epsilon}}\left(1-\frac{2 q_{\min }}{n\left|q_{-}\right|}+\frac{2 q_{\min }^{2}}{n^{2}\left|q_{-}\right|^{2}}+\frac{\omega^{2}}{2 E E^{\prime}}\right),
\end{aligned}
$$


which complies with the coherent bremsstrahlung spectrum in a straight crystal [6] (note that the dependence on $R$ drops out). However, due to the $\theta_{0}^{-2}$ dependence of (44), with the increase of $\left|\theta_{0}\right|$ to reach (41) the radiation intensity attenuates. Besides that, at large incidence angles the continuous potential approximation may be invalidated.

\section{CONDITIONS OF APPLICABILITY}

Our framework in the preceding two sections had been developed by the principle of maximal theoretical simplicity. In Sec. III] in our infinitesimal description of the particle deflection in the crystal we appealed to the high value of the particle energy. In Sec. IV we yet adopted the dipole approximation to radiation emission, which, however, is known [17] to break down at a sufficiently high energy. Therefore, we have to investigate whether these two approximations are mutually consistent under conditions of a real silicon crystal, and if yes, what is their compatibility domain. Yet, besides the continuous potential influence on the particle there exists incoherent scattering on individual nuclei, which affects the particle deflection as well as radiation. After all, in a case $R \gg\left|R_{c}\right|$ the condition of infinitesimal deflection certainly fails in vicinity of the volume reflection point, and that may also affect the radiation spectrum in some frequency domain. The present, last section comprises estimates of all the mentioned effects.

\section{A. Validity of the infinitesimal deflection approximation}

The condition of validity of the straight passage approximation is the smallness of the particle transverse displacement relative to the inter-planar distance. Based on Eq. (10), let us evaluate the particle transverse displacement as a function of time:

$$
\begin{gathered}
\triangle x(t)=\int_{-L / 2}^{t} d t^{\prime} \int_{-L / 2}^{t^{\prime}} d t^{\prime \prime} \frac{F\left(t^{\prime \prime}\right)}{E} \\
=\frac{2}{\pi R_{c}} \sum_{n=1}^{\infty} \frac{(-1)^{n} c_{n}}{n^{1+\epsilon}} \\
\cdot \int_{-L / 2}^{t} d t^{\prime} \int_{-L / 2}^{t^{\prime}} d t^{\prime \prime} \sin \left(2 \pi n \frac{b+\theta_{0} t^{\prime \prime}-\xi\left(t^{\prime \prime}\right)}{d}\right) .
\end{gathered}
$$

Changing here the order of integrations, and again expanding $\xi\left(t^{\prime \prime}\right)$ in Taylor series about point $t_{0}$, one converts the double integral in (45) to a single one which is

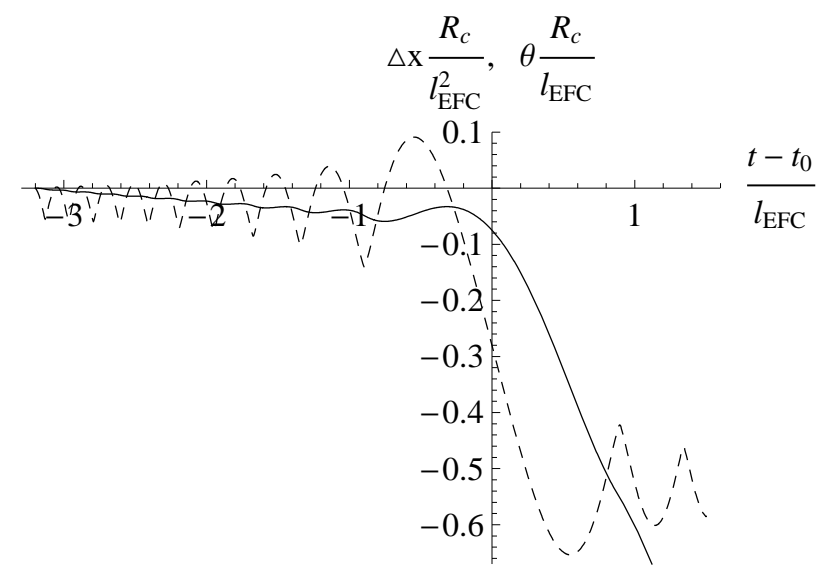

FIG. 8: The particle local transverse departure from the initial straight trajectory $\triangle x(t)$, expressed in units of $l_{\mathrm{EFC}}^{2} / R_{c}$ (solid line), and the local deflection angle $\theta(t)$, in units of $l_{\mathrm{EFC}} / R_{c}$ (dashed line), as functions of the distance to the point of tangency to crystalline bent planes (for some specific impact parameter). Both curves are built for the roomtemperature case $\epsilon=0.4$, crystal orientation (110) and for $\beta=-0.7$ (see Eq. (19)).

of Fresnel type:

$$
\begin{aligned}
\frac{\triangle x(t) R_{c}}{l_{\mathrm{EFC}}^{2}} & =\frac{2}{\pi} \sum_{n=1}^{\infty} \frac{(-1)^{n} c_{n}}{n^{1+\epsilon}} \\
\cdot & \int_{-\frac{L / 2+t_{0}}{l_{\mathrm{EFC}}}}^{\frac{t-t_{0}}{\mathrm{TFC}}} d \tau\left(\frac{t-t_{0}}{l_{\mathrm{EFC}}}-\tau\right) \sin \left\{2 \pi n\left(\beta-\tau^{2}\right)\right\} .
\end{aligned}
$$

It behaves as shown in Fig. 8 (by solid line). At large negative $\frac{t-t_{0}}{l_{\mathrm{EFC}}}$

$$
\begin{aligned}
& \frac{\triangle x(t) R_{c}}{l_{\mathrm{EFC}}^{2}} \underset{-\frac{t-t_{\mathrm{t}}}{l_{\mathrm{EFC}}} \gg 1}{\approx} \\
\approx & \left(1+\frac{t-t_{0}}{L / 2+t_{0}}\right) \\
& \frac{1}{2 \pi^{2}} \sum_{n=1}^{\infty} \frac{(-1)^{n-1} c_{n}}{n^{2+\epsilon}} \cos \left\{2 \pi n\left(\beta-\frac{\left(L / 2+t_{0}\right)^{2}}{l_{\mathrm{EFC}}^{2}}\right)\right\} \\
+ & \frac{l_{\mathrm{EFC}}^{2}}{8 \pi^{3}\left(t-t_{0}\right)^{2}} \sum_{n=1}^{\infty} \frac{(-1)^{n-1} c_{n}}{n^{3+\epsilon}} \sin \left\{2 \pi n\left(\beta-\frac{\left(t-t_{0}\right)^{2}}{l_{\mathrm{EFC}}^{2}}\right)\right\} .
\end{aligned}
$$

A curious feature here is the weak linear drift at the initial stage, visualized in Fig. 8 and represented in Eq. (47) by the term proportional to $1+\frac{t-t_{0}}{L / 2+t_{0}}$. It may be interpreted as a beam refraction at the entrance to the bent crystal. The refraction angle sign depends on the impact parameter at the entrance. However, by the absolute magnitude this effect is small, and for our current estimates less relevant.

The most relevant is the behavior of function (46) at $t>t_{0}$ where it grows linearly (which corresponds to a 
motion along the scattering angle final asymptote), as

$$
\begin{aligned}
& \frac{\triangle x(t) R_{c}}{l_{\mathrm{EFC}}} \underset{\frac{t-\tau_{0}}{l_{\mathrm{EFC}}} \gg 1}{\approx}\left(t-t_{0}\right) \frac{\sqrt{2}}{\pi} \sum_{n=1}^{\infty} \frac{(-1)^{n} c_{n}}{n^{3 / 2+\epsilon}} \sin \left(2 \pi n \beta-\frac{\pi}{4}\right) \\
& +\frac{1}{2 \pi^{2}} \sum_{n=1}^{\infty} \frac{(-1)^{n} c_{n}}{n^{2+n}} \cos \left\{2 \pi n\left(\beta-\frac{\left(L / 2+t_{0}\right)^{2}}{l_{\mathrm{EFC}}^{2}}\right)\right\} \\
& +\frac{l_{\mathrm{EFC}}^{2}}{8 \pi^{3}\left(t-t_{0}\right)^{2}} \sum_{n=1}^{\infty} \frac{(-1)^{n-1} c_{n}}{n^{3+\epsilon}} \sin \left\{2 \pi n\left(\beta-\frac{\left(t-t_{0}\right)^{2}}{l_{\mathrm{EFC}}^{2}}\right)\right\} \text {. }
\end{aligned}
$$

In fact, the acting force contribution builds up only before the particle reaches the final asymptote. For an actual estimate of the transverse displacement up to that moment one may simply take the value of (48) at $\frac{t-t_{0}}{l_{\mathrm{EFC}}} \simeq 1$. For reliability of the straight passage approximation, the corresponding transverse displacement $\Delta x$ needs to be less than the inter-planar interval half-width:

$$
\triangle x\left(t_{0}+l_{\mathrm{EFC}}\right) \ll d / 2 .
$$

With the use of Eq. (48), condition (49) boils down to

$$
R \ll \frac{\pi}{4}\left|R_{c}\right| . \quad \text { (for perturb. defl. angle) }
$$

This is physically obvious, since at $R \geq\left|R_{c}\right|$ nonperturbative effects such as planar channeling, or volume reflection in the bent crystal already become important.

Yet, for applicability of the stationary phase approximation the necessary requirement is that the external field coherence length $l_{\mathrm{EFC}}=\sqrt{2 R d}$ be small compared to the crystal half-thickness, i. e.,

$$
\sqrt{2 R d} \ll L / 2 .
$$

In what concerns applicability of the straight passage approximation to the radiation, the corresponding condition is milder than (49, 50). CBBC stems from the oscillatory part of the particle motion on the initial or the final asymptote, represented by last lines of Eqs. (477) and (48). Denoting that oscillatory part of motion as $\operatorname{var} \triangle x(t)$, the condition for the straight passage approximation applicability to the description of radiation is $\left.\operatorname{var} \triangle x\right|_{\left|t-t_{0}\right| \sim L / 2} \ll d / 2$. Substituting for var $\triangle x(t)$ the last line of (47) or (48), one arrives at the requirement

$$
\frac{L^{2}}{R^{2}} \gg \frac{2 \sqrt{2}}{\pi^{3}} \frac{d}{\left|R_{c}\right|} \text {. }
$$

That condition is essentially a product of (50) and (51), so when (51) holds very well, inequality (50) may be relaxed.

\section{B. Validity of the dipole approximation for radiation}

Secondly, we had employed dipole approximation for the radiation, which presumes smallness of the particle deflection angle compared to the typical radiation angle $\gamma^{-1}$. So, let us evaluate from Eq. (10) the local angle of deflection from the straight path. Using again the stationary phase approximation, one gets

$$
\begin{aligned}
& \theta(t)=\int_{-L / 2}^{t} d t^{\prime} \frac{F\left(t^{\prime}\right)}{E} \\
& \approx \frac{l_{\mathrm{EFC}}}{R_{c}} \frac{2}{\pi} \sum_{n=1}^{\infty} \frac{(-1)^{n} c_{n}}{n^{1+\epsilon}} \int_{-\infty}^{\frac{t-t_{0}}{l_{\mathrm{EFC}}}} d \tau \sin \left(2 \pi n\left(\beta-\tau^{2}\right)\right)
\end{aligned}
$$

This is an ordinary Fresnel integral; it is observed to converge within the range $l_{\mathrm{EFC}}$, and its asymptotic forms at $\left|t-t_{0}\right| \gg l_{\mathrm{EFC}}$ are [28]

$$
\begin{aligned}
& \theta(t) \underset{-\frac{t-t_{0}}{l_{\mathrm{EFC}}} \gg 1}{\approx} \frac{l_{\mathrm{EFC}}^{2}}{R_{c}\left(t_{0}-t\right)} \\
& \cdot \frac{1}{2 \pi^{2}} \sum_{n=1}^{\infty} \frac{(-1)^{n-1} c_{n}}{n^{2+\epsilon}} \cos \left(2 \pi n\left(\beta-\frac{\left(t-t_{0}\right)^{2}}{l_{\mathrm{EFC}}^{2}}\right)\right),
\end{aligned}
$$

and

$$
\begin{aligned}
& \theta(t)_{\substack{t-t_{0} \\
\tau_{\mathrm{EFC}}}}^{\underset{\mathrm{B}}{\approx}} \theta_{\mathrm{Born}} \\
& +\frac{l_{\mathrm{EFC}}^{2}}{R_{c}\left(t-t_{0}\right)} \frac{1}{2 \pi^{2}} \sum_{n=1}^{\infty} \frac{(-1)^{n-1} c_{n}}{n^{2+\epsilon}} \cos \left(2 \pi n\left(\beta-\frac{\left(t-t_{0}\right)^{2}}{l_{\mathrm{EFC}}^{2}}\right)\right) .
\end{aligned}
$$

Now, for validity in weakly bent crystals of the dipole approximation to radiation, we need smallness of the oscillatory part var $\gamma \theta(t)$ at $\left|t-t_{0}\right| \lesssim L / 2$. Substituting in Eq. (53) or the last line of Eq. (54) $\left|t-t_{0}\right| \rightarrow L / 2$, and replacing the sum by its typical value $1 / \sqrt{2}$, we get

$$
\left.\operatorname{var} \gamma \theta\right|_{\left|t-t_{0}\right| \lesssim L / 2} \ll 1 \Rightarrow \frac{L}{R} \gg \tilde{\theta}_{V}=\frac{2 \sqrt{2}\left|F_{1}\right| d}{\pi^{2} m} .
$$

Here $\tilde{\theta}_{V}$ is a parameter similar to $\theta_{V}=\frac{V_{0}}{m}$ of [17]. 229] With the use of parameters (9), numerically one finds

$$
\tilde{\theta}_{V} \approx 0.65 \cdot 10^{-4}
$$

both for Si (110) and Si (111). It is worth emphasizing that in a bent crystal the validity of the dipole approximation to radiation depends on $\tilde{\theta}_{V}$ smallness in comparison not with the Lindhard critical angle

$$
\theta_{c}=\sqrt{\frac{d}{2\left|R_{c}\right|}}
$$

(dependent on the particle energy via $R_{c}$ ), but with the active crystallographic plane bending angle $L / R$. 


\section{Influence of multiple scattering on particle deflection and on radiation}

\section{Deflection}

The angle of particle deflection in the continuous potential field also competes with the (rms, plane) angle of multiple scattering. The latter has a square root dependence on the medium thickness traversed [18]:

$$
\theta_{\text {mult }}(\triangle t)=\sqrt{\left\langle\theta_{x}^{2}\right\rangle_{\text {mult }}} \equiv \sqrt{\frac{1}{2}\left\langle\theta^{2}\right\rangle_{\text {mult }}}:=\frac{1}{\gamma} \sqrt{\frac{\triangle t}{l_{\text {mult }}}} .
$$

For electrons and positrons in silicon [18]

$$
l_{\text {mult }} \approx 0.13 \mathrm{~mm} . \quad\left(e^{ \pm} \text {in } \mathrm{Si}\right)
$$

For multiple scattering not to affect significantly the particle deflection in the target, $\theta_{\text {mult }}(L)$ must be less than the angle given by Eq. (17):

$$
\sqrt{\frac{L}{l_{\text {mult }}}} \ll \frac{\left|F_{1}\right| \sqrt{R d}}{m}
$$

which entails

$$
\frac{L}{R} \ll\left(\frac{F_{1}}{m}\right)^{2} l_{\text {mult }} d \approx\left\{\begin{array}{l}
3.6 \\
2.5
\end{array}\right\} \cdot 10^{-2} . \quad\left\{\begin{array}{l}
\mathrm{Si}(110) \\
\mathrm{Si}(111)
\end{array}\right\}
$$

\section{Radiation}

Concerning the coherent radiation at typical frequencies, again, condition (60) is not relevant. Instead, one is to compare $\theta_{\text {mult }}$ with angle $\operatorname{var} \theta(t)$ at $\left|t-t_{0}\right| \sim L / 2$. Should we be interested in the radiation angular distribution, $\theta_{\text {mult }}$ had to be count on the whole crystal thickness $L$. However, if only the (angle-integral) radiation spectrum is looked at, for absence of the multiple scattering influence on it, angle $\operatorname{var} \theta$ at $\left|t-t_{0}\right| \sim L / 2$ should be large compared to the multiple scattering angle only on the length $l_{\mathrm{EFC}}$ :

$$
\left.\operatorname{var} \theta\right|_{\left|t-t_{0}\right| \sim L / 2} \gg \triangle \theta_{\text {mult }}\left(l_{\mathrm{EFC}}\right)
$$

i. e.,

$$
\sqrt{\frac{l_{\mathrm{EFC}}}{l_{\text {mult }}}} \ll \tilde{\theta}_{V} \frac{R}{L}
$$

For the active crystalline plane bending angle this implies

$$
\frac{L}{R} \ll \tilde{\theta}_{V} \sqrt{\frac{l_{\mathrm{mult}}}{l_{\mathrm{EFC}}}} \approx\left\{\begin{array}{l}
1.7 \\
1.5
\end{array}\right\} \cdot 10^{-4}\left(\frac{1 \mathrm{~m}}{R}\right)^{1 / 4} \cdot\left\{\begin{array}{l}
\mathrm{Si}(110) \\
\mathrm{Si}(111)
\end{array}\right\}
$$

\section{Incoherent bremsstrahlung background}

Still another issue is that the coherent radiation receives a background from incoherent radiation acts. A standard way to estimate the incoherent bremsstrahlung intensity in a crystal is to take the radiation in an amorphous target made of the same material:

$$
\frac{d E_{\mathrm{BH}}}{d \omega}=\frac{L}{L_{0}}\left[\frac{4}{3}\left(1-\frac{\omega}{E}\right)+\frac{\omega^{2}}{E^{2}}\right] \Theta(E-\omega) .
$$

Here $L_{0}$ is the radiation length, for silicon amounting [18]

$$
L_{0}=9.36 \mathrm{~cm} \text {. }
$$

The $\omega$-dependence of (63) is mild, and as an estimate of $d E_{\mathrm{BH}} / d \omega$ one may take its value at $\omega \simeq 0$.

To compare with, the spectral intensity of the CBBC radiation at an average radiation frequency $\omega \sim \frac{\omega_{+}+\omega_{-}}{4}$ (see Figs. 6, 7) is about half of its maximal value (35):

$$
\begin{aligned}
\left.\frac{d E_{\mathrm{CBBC}}}{d \omega}\right|_{\omega \sim \frac{\omega_{+}+\omega_{-}}{2}} & \sim \frac{e^{2}}{3 \pi} \gamma^{2}\left\langle\theta_{\mathrm{Born}}^{2}\right\rangle \\
& \equiv \frac{2 e^{2} F_{1}^{2} R d}{3 \pi^{3} m^{2}} \sum_{n=1}^{\infty} \frac{c_{n}^{2}}{n^{3+2 \epsilon}} .
\end{aligned}
$$

$$
\left(\left|\theta_{0}\right|<L / 2 R\right)
$$

Numerically, Eq. (65b) gives

$$
\begin{aligned}
\frac{e^{2}}{3 \pi} \gamma^{2}\left\langle\theta_{\text {Born }}^{2}\right\rangle & =\frac{e^{2}}{3 \pi}\left(\frac{F_{1} d}{\pi m}\right)^{2} \frac{2 R}{d} \sum_{n=1}^{\infty} \frac{c_{n}^{2}}{n^{3+2 \epsilon}} \\
& \approx\left\{\begin{array}{c}
4.5 \cdot 10^{-4} \\
3 \cdot 10^{-4}
\end{array}\right\} \frac{R}{\mathrm{~cm}} . \quad\left\{\begin{array}{l}
\mathrm{Si}(110) \\
\operatorname{Si}(111)
\end{array}\right\}
\end{aligned}
$$

As had been mentioned at the end of Sec. [V] the coherent bremsstrahlung spectral intensity is independent of the crystal thickness $L$.

For CBBC radiation to manifest itself prominently, it must exceed the incoherent bremsstrahlung contribution:

$$
\frac{d E_{\mathrm{CBBC}}}{d \omega}>\frac{d E_{\mathrm{BH}}}{d \omega} .
$$

With (66. 63), it appears that the ratio $d E_{\mathrm{CBBC}} / d E_{\mathrm{BH}}$ depends only on the ratio $L / R$, i. e. on the active plane bending angle, with the proportionality coefficient

$$
\frac{d E_{\mathrm{CBBC}}}{d E_{\mathrm{BH}}} \sim 10^{-3} \frac{R}{L} .
$$

\section{E. Radiation at volume reflection (small $\omega$ domain)}

We had mentioned in Sec. VB that CBBC mechanism may be responsible for the generation of a large part of the radiation spectrum even when condition (50) is violated. In the latter case, the infinitesimal deflection 

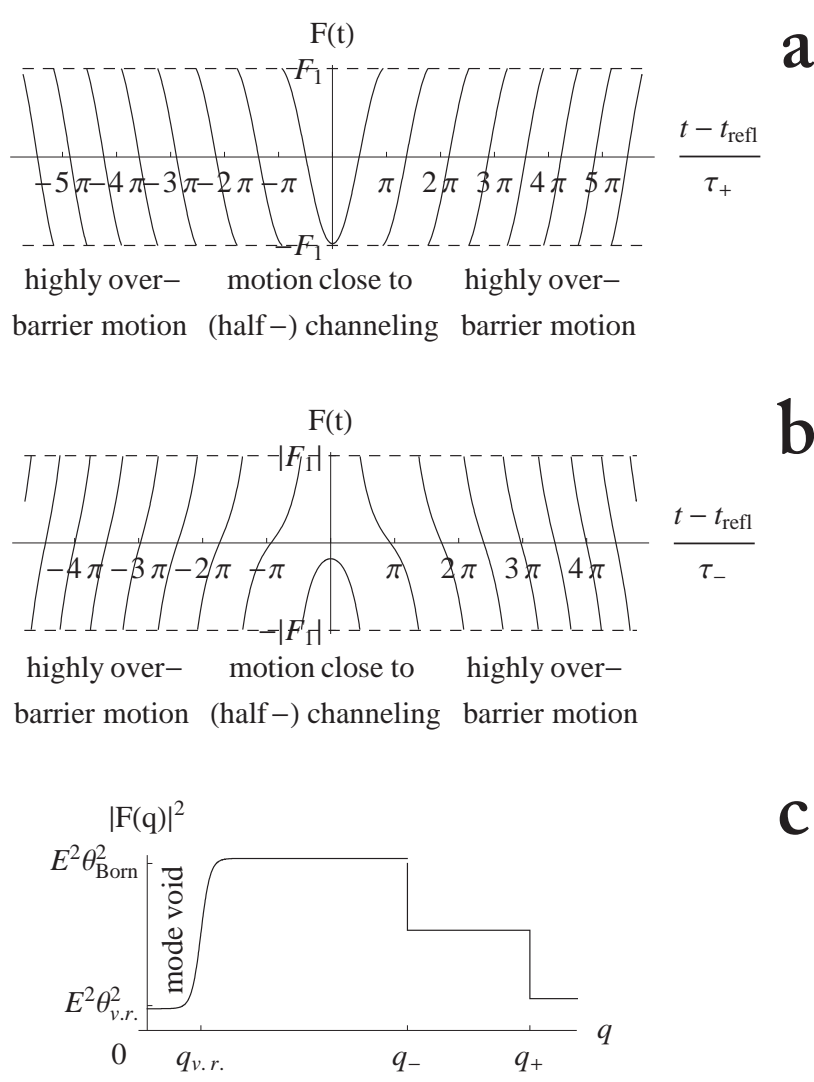

FIG. 9: (a) - exemplary graph of time-dependence of the force acting in a ((110) oriented, zero-temperature) bent crystal on a (positively) charged particle around the volume reflection point, at $R \gg R_{c}$ (the figure corresponds to $R=50 R_{c}$ ). The force discontinuities correspond to the particle passage through (sharp) potential maxima at the atomic plane positions. In vicinity of point $t=t_{\text {ref }}$ the trajectory draws nearly tangential to the maximum potential ridge, and in that sense is close to (half-) channeling. (b) - the same for negatively charged particles. The effective potential maximum is regular ( $F \rightarrow 0$ on top) and is situated approximately midway the atomic planes. (c) - schematic of the force Fourier transform modulus square. The dominant contribution to $|F(q)|^{2}$ and therethrough to $d E_{\mathrm{coh}} / d \omega$ comes from the interval $q_{\text {v.r. }} \leq q \leq q_{+}$.

approximation fails for evaluation of the particle final deflection angle, overestimating it, and hence the CBBC formula (33) must overestimate the radiation spectrum at sufficiently small $\omega$, where it is proportional to the final deflection angle squared. Let us now estimate the scale of $\omega$ at which modification of $\mathrm{CBBC}$ radiation is needed.

At $R \gg\left|R_{c}\right|$ the actual mechanism of particle deflection is volume reflection [3], whereat the magnitude of the deflection angle is of the order of Lindhard's critical angle (57). The contributing $q$-frequencies of particle oscillation during the volume reflection are effectively bounded from below [30] by the value equal to twice the channeling

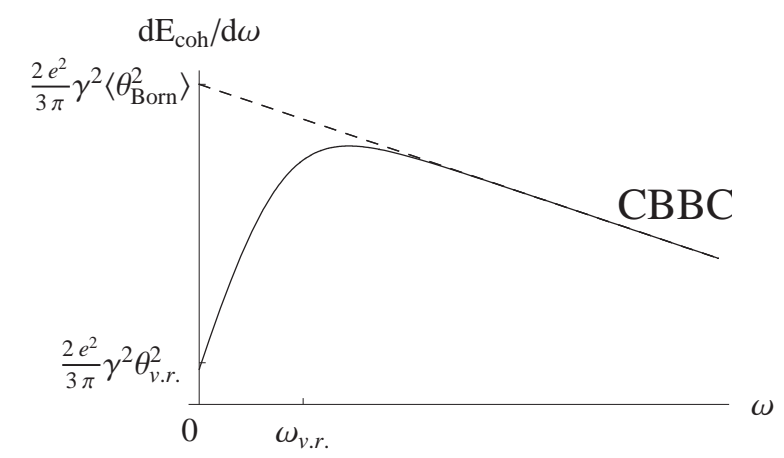

FIG. 10: Schematic of a turnover in the coherent radiation spectrum due to the volume reflection.

frequency $1 / \tau$ :

$$
q \geq q_{\text {v.r. }}=\frac{2}{\tau}
$$

with

$$
\begin{gathered}
\tau=\tau_{+}=\sqrt{\frac{2}{\left|R_{c}\right| d}} \quad \text { (pos. char. part.) } \\
\tau \simeq \tau_{-}=\tau_{+} \frac{\ln \frac{R}{\left|R_{c}\right|}}{2 \pi} \quad \text { (neg. char. part.) }
\end{gathered}
$$

(see Fig. 98). The mode void below frequency (69) arises because in the vicinity of the volume reflection point $t=t_{\text {refl }}$ (the closest approach to the axis of the crystal bending) the particle moves in each interval nearly by the channeling half period of the maximal amplitude, crossing the potential ridges at a nearly grazing angle (see Figs. 9a,b) [31]. As a consequence, at radiation frequency

$$
\omega_{\mathrm{v} . \mathrm{r} .}=\frac{1}{\frac{1}{E}+\frac{1}{2 \gamma^{2} q_{\mathrm{v} . \mathrm{r} .}}}
$$

the spectral intensity $d E_{\mathrm{coh}} / d \omega$ related with $|F(q)|^{2}$ through Eq. (22), must have a turnover (see Fig. 10), and drop at $\omega \rightarrow 0$ to $\frac{2 e^{2}}{3 \pi} \gamma^{2} \theta_{\text {v.r. }}^{2}$ (with $\theta_{\text {v.r. }}^{2}<\left\langle\theta_{\text {Born }}^{2}\right\rangle$, see Eq. (74) below) [16]. Hence, at $\omega \approx \omega_{\text {v.r. }}$ there forms a spectral maximum, or rather a "hump" feature, since at $\omega>\omega_{\text {v.r. }}$ the CBBC spectrum decreases rather slowly.

A broad maximum similar to the one shown in Fig. 10 had first been discovered in computer simulations [2, 4] of radiation at volume reflection, but its interpretation was not quite transparent. Now, we may conclude that as relative to $\mathrm{CBBC}$, the volume reflection effect on radiation is only of suppressive, not enhancing character. It stems from the particle inability to sustain in a strong inter-crystalline field a quasi-periodic motion at too low frequencies - the over-barrier particle can not spend in an inter-planar channel a time longer than the channeling period (actually, half period).

For the perturbative CBBC theory to have a significant applicability domain, frequency $\omega_{\text {v.r. }}$ must be much lower 
than the CBBC spectrum end-point $\omega_{+}$, which implies

$$
q_{\mathrm{v} . \mathrm{r.}} \ll \frac{\pi L}{R d}, \frac{m}{2 \gamma} .
$$

Condition $q_{\text {v.r. }} \ll \frac{m}{2 \gamma}$ with numerical values (9) translates into requirement

$$
E \ll 10 \mathrm{TeV},
$$

which is guaranteed by the present accelerator capabilities, whereas condition $q_{\text {v.r. }} \ll \frac{\pi L}{R d}$, basically, coincides with (52). The latter may also be regarded as a condition for the particle energy (see Eq. (82) below), which is, however, not very demanding. So, under the conditions of dipole radiation volume reflection effects should manifest themselves in a minor region of the spectrum, indeed.

It is yet relevant to estimate the relative depth of the volume reflection dip. The actual value of the volume reflection angle (in crystal with orientation (110)) is $\left|\theta_{\text {v.r. }}\right| \approx \frac{\pi}{2} \theta_{c}$ for positively charged, and $\left|\theta_{\text {v.r. }}\right| \approx \theta_{c}$ for negatively charged particles [19]. Therefore,

$$
\begin{aligned}
& \frac{d E_{\mathrm{v} . \mathrm{r} .}(0)}{d E_{\mathrm{CBBC}}(\simeq 0)}=\left\{\begin{array}{c}
(\pi / 2)^{2} \\
1
\end{array}\right\} \frac{\theta_{c}^{2}}{\left\langle\theta_{\text {Born }}^{2}\right\rangle} \approx\left\{\begin{array}{c}
6 \\
2.5
\end{array}\right\} \frac{\left|R_{c}\right|}{R} \text {. } \\
& \left\{\begin{array}{l}
\text { pos. char. part. } \\
\text { neg. char. part. }
\end{array}\right\}
\end{aligned}
$$

Thus, for the dip to be well discernible, one actually needs $R$ to be at least a few times larger than $R_{c}$.

\section{F. Crystal and beam optimal parameters}

Let us now assemble conditions (51, 52, 55, 62, 67) and examine their mutual compatibility, the variable parameters being $R$ and $L$. Eqs. (55, 62) together read

$$
\tilde{\theta}_{V} \ll \frac{L}{R} \ll \tilde{\theta}_{V} \sqrt{\frac{l_{\mathrm{mult}}}{l_{\mathrm{EFC}}}}
$$

which imply

$$
\sqrt{l_{\text {mult }} / l_{\mathrm{EFC}}} \ggg 1 \text {. }
$$

This is reminiscent of the Landau-Pomeranchuk-Migdal (LPM) condition

$$
l_{\text {form }} \ll l_{\text {mult }},
$$

but at typical radiation frequencies one estimates $l_{\text {form }} \lesssim$ $2 q_{ \pm}^{-1} \sim 2 \frac{R d}{\pi L}$, and according to inequality (51), it holds that $l_{\text {form }} \ll l_{\mathrm{EFC}}$. So, the LPM condition appears to be less crucial than (76).

For fulfilment of condition (76), with $l_{\text {mult }}$ fixed, one needs to have $l_{\mathrm{EFC}}$, i. e. $R$ and $d$, as low as possible. Note that the value of $d$ is lower for orientation (110) than for (111), thus orientation (110) is more beneficial. But as for $R$, at practice it is normally at least in the range of decimeters, which gives $\sqrt{l_{\text {mult }} / l_{\mathrm{EFC}}} \simeq 4$, while at highest $R \sim 10 \mathrm{~m}$ one has $\sqrt{l_{\mathrm{mult}} / l_{\mathrm{EFC}}} \simeq 1.4$. Thus, unfortunately, it is impossible to demand inequality (76) as really strong. Anyway, the optimal value for the active crystallographic plane bending angle is about

$$
\frac{L}{R} \sim \tilde{\theta}_{V}\left(\frac{l_{\mathrm{mult}}}{l_{\mathrm{EFC}}}\right)^{1 / 4} \sim 1.3 \cdot 10^{-4} . \quad \text { (optimal) }
$$

Then, the parameter of radiation non-dipoleness (also known as $\rho$-parameter [17]) is

$$
\left.\gamma \theta\right|_{\left|t-t_{0}\right| \sim L / 2} \sim \tilde{\theta}_{V} \frac{R}{L} \simeq 0.5,
$$

whereas the parameter of radiation decoherence due to multiple scattering is about the same:

$$
\frac{\triangle \theta_{\text {mult }}\left(l_{\mathrm{EFC}}\right)}{\left.\theta\right|_{\left|t-t_{0}\right| \sim L / 2}} \sim \frac{R}{\tilde{\theta}_{V} L} \sqrt{\frac{l_{\mathrm{EFC}}}{l_{\text {mult }}}} \approx 0.5 .
$$

In view of the narrowness of condition (76), it seems reasonable to suggest that since the size of the coherence length remains the same for all locations within a uniformly bent crystal, and the length $l_{\text {mult }}$ is constant as well, then even if condition (62) fails (multiple scattering effects are substantial), the spectrum shape may still be roughly described by the present theory, only the intensity being suppressed by a factor depending on the ratio $l_{\mathrm{EFC}} / l_{\text {mult }}$. However, evaluation of such a factor is beyond the scope of the present article.

Within our framework, presuming all the abovementioned conditions to be fulfilled, let us check the last crucial condition (67). With (78), ratio (68) will amount $d E_{\mathrm{CBBC}} / d E_{\mathrm{BH}} \sim 7$, which is satisfactorily high.

Other relevant conditions (51, 52) are rather easy to fulfil. At bending angle (78) Eq. (51) demands for the crystal thickness

$$
\frac{L}{d} \gg \frac{8 R}{L} \approx 6 \cdot 10^{4} \Rightarrow L \gg\left\{\begin{array}{l}
12 \\
20
\end{array}\right\} \mu \mathrm{m} . \quad\left\{\begin{array}{l}
\mathrm{Si}(110) \\
\mathrm{Si}(111)
\end{array}\right\}
$$

Eq. (52) together with (78) sets the lower bound for the electron energy:

$$
E \gg \frac{2 \sqrt{2}}{\pi^{3}} \frac{R^{2}}{L^{2}}\left|F_{1}\right| d \sim 1 \mathrm{GeV} .
$$

Towards experimental investigation of CBBC itself, let us yet determine the full set of parameters at which CBBC effects are least deteriorated and the sharpest. As we had mentioned, lower $R$ are favored for cleaner CBBC, but $R$ is tightly interrelated with $L$, which must satisfy inequality (81). Taking, marginally

$$
L_{\star} \approx\left\{\begin{array}{l}
50 \\
80
\end{array}\right\} \mu \mathrm{m} \quad\left\{\begin{array}{l}
\mathrm{Si}(110) \\
\mathrm{Si}(111)
\end{array}\right\}
$$

(such thin samples are available - cf. Ref. 1 of [10]), entails through Eq. (78)

$$
R_{\star} \approx\left\{\begin{array}{l}
0.4 \\
0.6
\end{array}\right\} \mathrm{m} . \quad\left\{\begin{array}{l}
\mathrm{Si}(110) \\
\mathrm{Si}(111)
\end{array}\right\}
$$


For what concerns the electron energy, to avoid a pronounced volume reflection turnover one needs, according to (74), smaller ratio $R /\left|R_{c}\right|$, i. e. higher $E$. Besides that, if one wants the "ankle" features in the radiation spectrum not to be smeared out by the photon recoil effects (cf. Figs. 6]and 7), one should arrange the condition $\frac{2 \pi L \gamma^{2}}{R d} \ll E$, which under (78) implies

$$
E \ll \frac{m^{2} d}{2 \pi} \frac{R}{L} \approx\left\{\begin{array}{l}
300 \\
500
\end{array}\right\} \mathrm{GeV} . \quad\left\{\begin{array}{l}
\mathrm{Si}(110) \\
\mathrm{Si}(111)
\end{array}\right\}
$$

If in marginal fulfilment of (85) one takes

$$
E_{\star} \approx 150 \mathrm{GeV}
$$

we derive

$$
\left|R_{c}\right|_{\star} \sim\left\{\begin{array}{l}
25 \\
37
\end{array}\right\} \mathrm{cm}, \quad\left\{\begin{array}{l}
\mathrm{Si}(110) \\
\mathrm{Si}(111)
\end{array}\right\}
$$

whereby $R_{\star} /\left|R_{c}\right|_{\star} \approx 1.6$, and according to Eq. (74), the dip should not develop. Parameters (83, 84, 86, are "ideal" for checking the calculations in the present paper; the spectrum thereat must look like that in Fig. (6). The $\omega$ range is up to $\frac{1}{\frac{1}{E}+\frac{R d}{2 \pi \gamma^{2} L}} \sim 35 \div 50 \mathrm{GeV}$ and the expected spectral intensity maximum is $\frac{d E_{\mathrm{CBBC}}(0)}{d \omega} \approx 0.04$. The most demanding condition seems to be the initial electron beam collimation degree $\delta \theta_{0}<\frac{L}{2 R} \approx 70 \mu \mathrm{rad}$. If not achieved, an averaging of the radiation spectrum over the electron beam incidence angles must be performed.

\section{SUMMARY AND DISCUSSION}

The present study substantiates the notion that spectral intensity of radiation from ultra-high-energy electrons and positrons in a bent crystal is a sum of contributions from particle motion intervals on which the local angle between the particle velocity and the bent crystalline planes is definite, and so each such contribution is similar to coherent bremsstrahlung in a straight crystal. The length of an elementary coherence interval is $\simeq l_{\mathrm{EFC}}=\sqrt{2 R d}$, implying that (i) the crystal must be much thicker than the mentioned value (see Eq. (51)); (ii) the radiation spectral intensity (Eqs. (30,33)) is proportional to $l_{\mathrm{EFC}}^{2} \propto R d$, and does not depend on the crystal thickness. Still, under the "moderately high energy" condition (38) the crystal thickness determines the spectrum extent, and therethrough, the total energy emitted.

The characteristic feature of CBBC from a single electron is the well-defined end of the radiation spectrum, whose position depends on the active crystallographic plane bending angle $L / R$. At an angle of electron incidence on the crystal comparable to $\frac{L}{2 R}$ this end of spectrum splits into a pair of breaks (see Fig. 6). That feature must in principle be experimentally verifiable with a sufficiently well collimated initial beam $\left(\delta \theta_{0}<\frac{L}{2 R}\right)$. The best experimental conditions for investigating CBBC were described in Sec. VF.

We have also qualitatively discussed the modification of the coherent radiation spectrum in the domain of small $\omega$ owing to the onset of the volume reflection phenomenon possible when $R \gg\left|R_{c}\right|$. That modification is of purely suppressive character and manifests itself as a dip at the beginning of the spectrum. Next to the dip, around frequency (71) there appears to be a maximum in the spectrum, but it is not to be interpreted as a resonance.

The theoretical description adopted in this article had resorted to many simplifications - it did not properly incorporate the temperature dependence of the potential, neglected multiple scattering, and relied on an infinitesimal approximation to the particle deflection (in the bulk of the medium) as well as on dipole description of the radiation. Conditions (51, 78, 82) under which those approximations hold, altogether appear to be restrictive for the crystal bending angle (see Eq. (78) ), so generalization to a non-dipole treatment, and an account of the multiple scattering would be highly desirable. Nonetheless, let us mention that the dipole $\mathrm{CBBC}$ conditions are quite nicely met, e. g., in recent experiment [2]. Comparison of the CBBC theory with the available experimental data is intended elsewhere.

In conclusion, let us remark that although our paper presumed dependence of the crystal deformation only on one, longitudinal, coordinate, in principle higherdimensional deformation cases are conceivable, emerging under application of torsion, or owing to intrinsic crystal mosaicity. In those cases the stationary phase approximation must still be applicable, but the description should inevitably become more sophisticated.

Acknowledgement. The author wishes to thank A. V. Shchagin for fruitful discussions.
[1] A. G. Afonin et al., JETP Lett. 88 (2008) 414.

[2] W. Scandale et al., Phys. Rev. A 79 (2009) 012903.

[3] A. M. Taratin and S. A. Vorobiev, NIMB 26 (1987) 512.

[4] Yu. A. Chesnokov, V. I. Kotov, V. A. Maisheev, and I. A. Yazynin, JINST 3 (2008) P02005.

[5] V. A. Arutyunov, N. A. Kudryashov, V. M. Samconov, and M. N. Strikhanov, Nucl. Phys. B 363 (1991) 283.

[6] G. Diambrini Palazzi, Rev. Mod. Phys. 40 (1968) 611; M. L. Ter-Mikayelyan. High Energy Electromagnetic Pro- cesses in Condensed Media, Wiley, New York, 1972.

[7] V. G. Baryshevsky, I. Ya. Dubovskaya, and A. O. Grubich, Phys. Lett. A 77 (1980) 61; V. V. Kaplin, S. V. Plotnikov, and S. A. Vorob'ev, Zh. Tekh. Fiz. 50 (1980) 1079; S. Belucci et al., Phys. Rev. ST 7 (2004) 023501; A. V. Korol, A. V. Solov'yov, and W. Greiner, Int. J. Mod. Phys. E 13 (2004) 867; N. F. Shul'ga, V. V. Boyko, and A. S. Esaulov, Phys. Lett. A 372 (2008) 2065. 
[8] S. Bellucci et al., Phys. Rev. Lett. 90 (2003) 034801.

[9] Yu. M. Ivanov et al., JETP Lett. 81 (2005) 99.

[10] V. Guidi, A. Mazzolari, D. De Salvador, and A. Carnera, J. Phys. D 42 (2009) 182005; S. G. Lekhnitskii. Theory of Elasticity of an Anisotropic Body. Mir, Paris, 1981.

[11] J. Lindhard, Mat. fys. medd. Kgl. Danske vid. Selskab. 34 (1965) 14.

[12] V. M. Biryukov, Yu. A. Chesnokov, and V. I. Kotov, Sov. Phys. Usp. 37 (1994) 937.

[13] E. N. Tsyganov, Fermilab Report No. TM-682, 1976 (unpublished); Fermilab Report No. TM-684, 1976 (unpublished).

[14] F. W. J. Olver. Asymptotics and Special Functions. Academic Press, New York, 1974.

[15] T. M. Apostol. Introduction to Analytic Number Theory. Springer, New York, 1976.

[16] L. D. Landau and E. M. Lifshitz. The Classical Theory of Fields. Pergamon, London, 1962.

[17] V. N. Baier, V. M. Katkov, and V. M. Strakhovenko. Electromagnetic processes at high energies in oriented single crystals. World Scientific, Singapore, 1998.

[18] B. Rossi. High Energy Particles. Prentice-Hall, New York, 1952; C. Amsler et al. [Particle Data Group], Phys. Lett. B 667 (2008) 1.

[19] M. V. Bondarenco, arXiv:0911.0107.

[20] In experiments [1, 2] it takes on values $3.5 \theta_{c}$ and $6 \theta_{c}$, which is rather large.

[21] In [9] it has been asserted that this technique fails with (110) planar orientation, but works best with (111). Thus, we find it necessary in our article to discuss cases of (110) and (111) in parallel.
[22] In cases when the active crystal plane bending is achieved due to secondary elastic (quasimosaic) effects, the quadratic approximation for $\xi$ may be even more accurate than that of a circular arc. The author acknowledges communication with $\mathrm{V}$. Guidi on this point.

[23] Taking $V_{L} \approx 26.5 \mathrm{eV}, V_{S} \approx 7.5 \mathrm{eV}$, one obtains $c_{2}=$ $-0.9, c_{3}=-1.7, c_{4}=-2.2, c_{5}=-1.4$.

[24] Thereby, it can also be regarded as first Born approximation in coupling with the crystalline field.

[25] They coalesce into one in the limit $\theta_{0} \rightarrow 0$.

[26] Eq. (34) results as a solution to equation $q_{\min }\left(\omega_{ \pm}\right) \equiv$ $\frac{m^{2} \omega_{ \pm}}{2 E\left(E-\omega_{ \pm}\right)}=\left|q_{ \pm}\right|$.

[27] Which is sometimes also referred to as coherence length (in the sense of coherence in the process of photon emission), hence possible confusion.

[28] It is evident that (53) is basically a derivative of (47).

[29] For a parabolic potential, $V_{0}=\left|F_{1}\right| d / 4$, which implies $\tilde{\theta}_{V}=\frac{8 \sqrt{2}}{\pi^{2}} \theta_{V} \approx 1.1 \theta_{V}$. Thus, the difference between the definitions $\theta_{V}$ and $\tilde{\theta}_{V}$ is inessential.

[30] Apart from a small, nearly constant contribution from the finite total deflection angle.

[31] The particle also makes one nearly full undulation period, containing the point $t=t_{\text {refl }}$, but alone it can not give radiation competing with coherent radiation from several half-periods. Rather, this single nearly full period may be regarded as transition from one semi-channeled motion to another, standing in antiphase, and no interference of radiation from such antiphased trajectory parts being possible, despite their identical periods. 